\title{
Tasting the Bitter Sunlight
}

Jami L. Milton | doi:10.1371/journal.pbio.0060205

Have you ever had that gut reaction to your surroundings, some physical sensation that something isn't quite right? Maybe a squirmy, uneasy feeling in your stomach or an acrid taste on your tongue that makes you want to leave the scene? When the nematode C. elegans encounters an offensive sensation-whether a pungent, potentially dangerous odor (such as those associated with fungal parasites), extreme temperature, or the poking probe of a researcher-it wastes no time in making a hasty retreat. Researchers have elucidated the mechanisms of avoidance in these tiny worms to remarkable molecular and cellular detail (a much clearer picture than the often mysterious avoidance behaviors of humans).

In a new study, Stacey L. Edwards, Kenneth G. Miller, and colleagues expand the level of our understanding by describing a striking new behavior and mechanism of sensory detection for these microscopic animals. They demonstrate that short-wavelength light, such as blue-violet and ultraviolet (UV), is a potent avoidance cue, stimulating a robust acceleration of locomotion when the tail or body is illuminated, while head illumination drives reversal locomotion. After identifying and characterizing this new behavior, Edwards et al. used classic forward genetics (whereby animals are mutagenized and screened for defective phenotypes) to identify mutants that are unresponsive to UV light. Surprisingly, the mutations mapped to a gene that encodes a protein unrelated to any of the known phototransduction systems in nature. The authors named this new protein LITE-1 and showed that it functions as a UV light receptor. Interestingly, LITE-1 is related to the large family of insect Gustatory receptors that mediate taste responses. The closest homology is to that of fly Gustatory receptors, which detect water-soluble sugars and carbon dioxide.

The identification of a Gustatorylike receptor as a photoreceptor is unprecedented, and the skeptical mind might first question this finding.

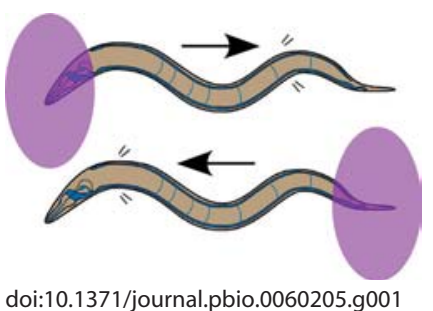

The C. elegans photophobic response to short-wavelength light is mediated by the LITE-1 ultraviolet light receptor. Here, an adult nervous system is highlighted in blue. Violet ovals represent illumination of the head or tail regions with a low-power, blueviolet laser beam, and arrows depict the direction of movement.

Perhaps the worms are responding to the heat generated by shortwavelength light? The authors rule out this possibility by using high powers of green light (to which worms show no response) to show that there is no correlation between light-induced temperature increases and the response of the animals. Other skeptics may question why $C$. elegans needs to detect UV light, especially given the common lore that it is a subterranean soil organism. However, the authors cite recent studies suggesting that $C$. elegans may spend much of its time above ground, hitching a ride on insects. Since a sunburn on an animal that is only a few cells thick would be lethal, the robust response of C. elegans to short wavelength light may be a hard-wired survival mechanism for avoiding direct sunlight. Indeed, the authors demonstrated that the UV light in direct sunlight is sufficient to elicit photophobic behavior.

The authors reasoned that a persuasive experiment fingering LITE-1 as a photoreceptor would be to introduce the gene into tissues that normally do not respond to light to see if they could elicit an ectopic light response. Accordingly, they expressed lite- 1 cDNA in muscle cells, including the vulval muscles that mediate egg laying. The result produced novel, light-driven muscle contractions, and in the case of the vulval muscles, initiated an egg laying response. Edwards et al. also forced lite-1 expression in all neurons of lite- 1 mutants, and in a specific subset of motor neurons that express the neurotransmitter acetylcholine. They also used regional illumination with a laser beam to activate LITE-1 just in tail neurons. In all cases, the researchers succeeded in rescuing UV light responses. These results narrow the playing field for understanding exactly which neurons mediate the UV response, suggesting that a small subset may be responsible. While the precise identities of the endogenous LITE-1-carrying neurons remain elusive, future studies can focus on these subsets.

Animals, including worms, rely on communication between motor neurons and muscles to drive locomotion. Neurons mediate locomotion behavior in part by engaging $\mathrm{G}$ proteins, which are molecular switches that are universally important for neuronal communication and the release of chemical messengers between neurons. Worms containing mutations in either of two major $\mathrm{G}$ protein pathways are paralyzed and fail to respond to harsh stimuli. But by exposing just the tail (or the whole body) to UV light, Edwards et al. were able to reverse this paralysis and restore coordinated locomotion. This bizarre and startling resurrection of normally immobilized animals gives the scientists insight into how LITE-1 is working, and suggests that the LITE-1 pathway overrides classical G protein signaling.

Like most seminal work, the curious identification of a Gustatory-like receptor family protein behaving as a light-sensing molecule opens a wave of new directions for understanding this novel photophobic behavior and photoreceptors. As the authors point out, it may be possible to harness this remarkable sunlight-tasting protein as a tool to produce specific light-activated responses in cells or living animals.

\footnotetext{
Edwards SL, Charlie NK, Milfort MC, Brown BS, Gravlin CN, et al. (2008) A novel molecular solution for ultraviolet light detection in Caenorhabditis elegans. doi:10.1371/journal.pbio.0060198
} 